\title{
Analytical model of a SAW gas sensor
}

\author{
M. Urbańczyk \\ Faculty of Electrical Engineering, \\ Silesian University of Technology, Poland
}

\begin{abstract}
SAW gas sensors are attractive because of their remarkable sensitivity due to changes of the boundary conditions (mechanical and electrical in the acoustoelectric effect) propagating of the Rayleigh wave, introduced by the interaction of a thin chemically active sensor film with gas molecules. This unusual sensitivity results from the fact that most of the acoustic wave energy is concentrated near the waveguide surface within approximately one or two wavelengths. In the paper a new theoretical model of analysing a SAW gas sensor is presented. The effect of SAW velocity changes depends on the profile concentration of diffused gas molecules in the porous sensor film. Basing on these analytical results, the sensor structure can be optimized. Some numerical results are shown.
\end{abstract}

Keywords: gas sensor, SAW, acoustoelectric effect, Knudsen's diffusion in porous film, numerical modelling.

\section{Introduction}

A very interesting feature of SAW sensors is the fact that a layered sensor structure on a piezoelectric substrate provides new possibilities of detecting gas making use of the acoustoelectric coupling between the Rayleigh waves and the free charge carriers in the semiconductor sensor layer. Using a configuration with a dual delay line and an adequately chosen active layer, a sensor with a high sensitivity and good temperature stability can be designed [1, 2]. SAW gas sensors are attractive because of their remarkable sensitivity due to changes of the boundary conditions of propagating the surface wave. This unusual sensitivity results from the simple fact that most of the acoustic wave energy is concentrated near the crystal surface within approximately one or two wavelengths. 
Any change of the physical properties of the thin active layer placed on a piezoelectric substrate can affect the SAW propagation. However, from the practical point of view, only the following two affects are potentially significant, namely a change in the mass of the layer and a change of its electrical conductivity, which cause a significant change in the velocity and attenuation of SAW. These two effects occur simultaneously. In semiconductor layers the electric effect is much greater (several times, depending on the gas concentration) [2].

In order to optimize the structure of the sensor it is important to get an analytical model of the SAW sensor. This paper provides theoretical considerations concerning the acoustoelectric effect in the piezoelectric acoustic delay line and semiconducting sensor layer configuration. This will be the starting point to construct a multilayer analytical model. The sensor layer with absorbed gas molecules may be treated as a stack of partial layers with a different electrical conductivity due to the concentration profile of gas molecules in the sensor layer. These partial layers are positioned at different distances from the piezoelectric surface.

The paper summarizes the acoustoelectric theory, i.e. Ingebrigtsen's formula [3], the impedance transformation law, gas concentration profiles, and predicts the influence of a thin semiconductor sensor layer on the SAW velocity. In the paper a new theory of analyzing a SAW gas sensor is proposed. Optimizing this structure, we obtain a high sensitivity of specific gas detection. In order to reach a high sensitivity we must determine the thickness and porosity of the layer for the given gas molecules, the temperature of operation, and other parameters.

\section{Acoustoelectric effect in the piezoelectric-semiconductor layer}

In the case of small disturbances, both mass and the electrical "load" may be considered separately. The total effect of a relative change of the wave vector $\Delta k / k_{0}$ and the velocity of propagation $\Delta v / v_{0}$ is the sum of both these components [4]:

$$
\frac{\Delta k}{k_{0}} \approx\left(\frac{\Delta k}{k_{0}}\right)_{m}+\left(\frac{\Delta k}{k_{0}}\right)_{\sigma}
$$

By means of the perturbation analysis the contribution of each of these effects can be determined. We assume that the mass of the sensor layer may be neglected. Further on only the electric effect will be taken into consideration.

\subsection{Electrical surface perturbations}

If the electrical boundary conditions are perturbed only at the upper surface $y=0$, the perturbation formula is [5]:

$$
(\Delta k)_{\sigma} \approx \frac{\omega\left[\varphi^{\prime}(0) D_{y}^{*}(0)-\varphi^{*}(0) D_{y}^{\prime}(0)\right]}{4 P}
$$

where the index $\sigma$ refers to the change of the wave number due to electrical surface perturbations. 
Unperturbed electrical boundary conditions are usually neither short-circuit $[\varphi(0)=0]$ nor open-circuit $\left[D_{y}(0)=0\right]$ ones, and it is not clear whether the potential or the electrical displacement should be approximated by its unperturbed value. The best agreement between the perturbation theory and exact numerical calculations is obtained by using the so-called weak-coupling approximation, in which the stress field $T$ is assumed to be unchanged by the perturbation $[3,5]$. It is most convenient to express the electrical boundary conditions in terms of the electrical surface impedance per unit area:

$$
Z_{E}(0)=\left(\frac{\varphi}{i \omega D_{y}}\right)_{y=0}
$$

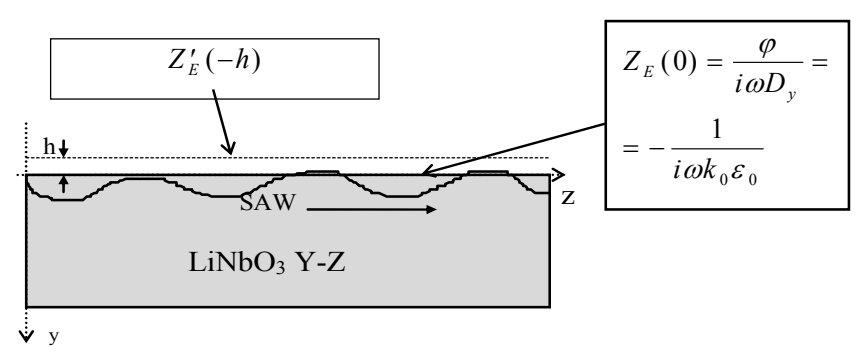

Figure 1: Electrical boundary conditions.

Let us assume that unperturbed electrical boundary conditions correspond to a free substrate surface, i.e. the region above the substrate $(y<0$ in fig. 1$)$ is a vacuum and extends to $\mathrm{y} \rightarrow \infty$. The space-charge potential satisfying the Laplace equation is reduced in this region to the form:

$$
\nabla^{2} \Phi=0
$$

The unperturbed potential function is therefore:

$$
\Phi=\Phi_{R}(y) e^{-i k z}=e^{k y} e^{-i k z}, \mathrm{y}<0
$$

and the normal component of electrical displacement is:

$$
D_{y}=-k \varepsilon_{0} e^{k y} e^{-i k z}, \mathrm{y}<0
$$

Consequently, the unperturbed surface impedance is:

$$
Z_{E}(0)=-\frac{1}{i \omega k_{0} \varepsilon_{0}}
$$

The perturbed normalized surface impedance is:

$$
z_{E}^{\prime}(0)=\frac{Z_{E}^{\prime}(0)}{\left|Z_{E}(0)\right|}=-i k_{0} \varepsilon_{0}\left(\frac{\varphi^{\prime}}{D_{y}^{\prime}}\right)_{y=0}
$$

where ' indicates perturbed quantities. The potential $\varphi$ ' $(0)$ of the perturbed fields and electrical displacement $D_{y}$ ' $(0)$ are now related to the unperturbed fields:

$$
\begin{aligned}
& \varphi^{\prime}(0)=\varphi(0)+A \\
& D_{y}^{\prime}(0)=D_{y}(0)+k_{0} \varepsilon_{p}^{T} A
\end{aligned}
$$


where $\varepsilon_{p}^{T}=\sqrt{\varepsilon_{y y}^{T} \varepsilon_{z z}^{T}-\left(\varepsilon_{y z}^{T}\right)^{2}}$ and $k_{0}{ }^{\prime}=k_{0}$ has been approximated.

Now the perturbed potential $\varphi^{\prime}$ and electrical displacement $D^{\prime}{ }_{y}$ on the surface may be expressed by the formula:

$$
\begin{gathered}
\varphi^{\prime}(0)=-i z_{E}^{\prime}(0) \frac{\left(\varepsilon_{0}+\varepsilon_{p}^{T}\right)}{\varepsilon_{0}-i \varepsilon_{p}^{T} z_{E}^{\prime}(0)} \varphi(0) \\
D_{y}^{\prime}(0)=-\frac{k_{0} \varepsilon_{0}\left(\varepsilon_{0}+\varepsilon_{p}^{T}\right)}{\varepsilon_{0}-i \varepsilon_{p}^{T} z_{E}^{\prime}(0)} \varphi(0)
\end{gathered}
$$

Finally, we obtain:

$$
\frac{\Delta k}{k_{0}}=-\left(\frac{\Delta v}{v_{0}}\right)_{s c} \frac{1+i z_{E}^{\prime}(0)}{1-i \frac{\varepsilon_{p}^{T}}{\varepsilon_{0}} z_{E}^{\prime}(0)}=\frac{K^{2}}{2} \frac{1+i z_{E}^{\prime}(0)}{1-i \frac{\varepsilon_{p}^{T}}{\varepsilon_{0}} z_{E}^{\prime}(0)}
$$

$K^{2}=2\left(\frac{\Delta v}{v_{0}}\right)_{s c}$ - the electromechanical coupling factor, and the index $s c$ indicate perturbation due to an electrical short circuit on the boundary. Eq. (12) is the Ingebrigtsen formula for electrical surface perturbations.

\subsection{Thin semiconducting sensor layer}

In the case of the semiconducting layer the surface impedance may be determined considering the motion of the charge carriers in the layer. Let us assume that the semiconductor layer on the piezoelectric substrate is thinner than a Debye length. In the one-dimensional case the equation of the current density in the direction $z$ (fig.1) is expressed as [6]:

$$
I_{z}=\rho_{01} \mu E_{z}-D \frac{d \rho_{1}}{d z}
$$

where $\rho_{01}$ and $\rho_{l}$ are the intrinsic and whole charge density per unit length, respectively. The quantities $\mu$ and $D$ are the carrier mobility and diffusion quantity, respectively, $E_{z}$ - electric intensity field in the direction $z$. For the time dependence of the $E_{z}$ expressed as $\exp i(\omega t-k z)$ the continuity equation [8], concerning current density in the sensor layer is:

$$
\frac{d I_{z}}{d z}+i \omega \rho_{l}=0
$$

The surface density of charge carriers $\rho_{s}=\rho_{l} / a(a-$ width of the layer $)$ induced in the layer by the electric field $E_{z}$ will be:

$$
\rho_{s}=\frac{\left(\frac{\omega_{c}}{\omega}\right) \varepsilon k h}{1-i \frac{\omega}{\omega_{D}}} E_{z}
$$

where: $\omega_{c}=\frac{\mu\left|\rho_{0}\right|}{\varepsilon}$ relaxation frequency, $\omega_{D}=\frac{v_{0}^{2}}{D}=\frac{k_{B} T}{q} \mu$ diffusion frequency, $k_{B^{-}}$ Boltzman constant, $T$ - temperature, $q$ - electric charge. 
From the boundary conditions of the space-charge field in a single semiconductor layer in the plane $y=0$ results the continuity of the potential $\varphi\left(0^{-}\right)$ $=\varphi\left(0^{+}\right)$and the condition of dielectric displacement $D_{y}\left(0^{-}\right)-D_{y}\left(0^{+}\right)=\rho_{s}$, where $D_{y}\left(0^{-}\right)=\varepsilon_{0} E_{y}\left(0^{-}\right)$, and assuming the electrostatic condition we can write that $E_{z}^{-}=$ $i k_{0} \varphi(y, z)$. By applying Gauss' law and the potential continuity in the plane $y=0$, the impedance $z_{E}^{\prime}(0)$ can be expressed in the following form:

$$
z_{E}^{\prime}(0)=\left(-i+\frac{\frac{\sigma_{s}}{\varepsilon_{0} v_{0}}}{1-\mathrm{i} \frac{\omega}{\omega_{\mathrm{D}}}}\right)^{-1}
$$

where $\sigma_{s}=\sigma h$ is the surface conductivity of the layer.

In semiconducting sensor layers the diffusion effect is usually very small $(D \sim 0)$ and the surface conductivity is independent of the frequency of the wave propagation. In this case the surface impedance $z_{E}^{\prime}(0)$ is only a function of the surface conductivity $\sigma_{s}$ :

$$
z_{E}^{\prime}(0)=\left(-i+\frac{\sigma_{s}}{\varepsilon_{0} v_{0}}\right)^{-1}
$$

\section{Profile of the gas concentration in the sensor layer}

In gas sensors the effect of SAW velocity changes vs. the surface conductivity depends on the profile of concentration of diffused gas molecules in the porous film. The mechanism of gas diffusion through a porous material depends on the size of the pores and the type of surface diffusion. In porous sensing material usually Knudsen's diffusion is assumed. This type of diffusion depends on the pore radius and prevails in pores ranging from 1 to $100 \mathrm{~nm}$ in radius [7]. The Knudsen diffusion constant, $D_{K}$, depends on the molecular weight of the diffusing gas, $M$, the pore radius, $r$, temperature, $T$, and the universal gas constant, $R$, as follows:

$$
D_{K}=\frac{4 r}{3} \sqrt{\frac{2 R T}{\pi M}}
$$

If the pore radius $r>2 \mathrm{~nm}$, a free molecular flow exists. That means that the gas molecules collide more frequently with the boundaries than with other gas molecules. The original studies of a free-molecule flow were limited to small holes in very thin plates. Gas molecules are consumed rapidly or slowly due to the surface reaction in the sensing layer [7].

Let us consider the gas diffusion in a porous thin semiconducting film, as shown in fig. 2.Two assumptions, i.e. Knudsen diffusion and first-order surface reaction, allow to formulate the well-known diffusion equation [7, 9]:

$$
\frac{\partial C_{A}}{\partial t}=D_{K} \frac{\partial^{2} C_{A}}{\partial x^{2}}-k C_{A}
$$

where $C_{A}$ - is the concentration of target gas, $t$ - time, $D_{K}$ - Knudsen diffusion constant, $y$ distance from the bottom layer, counted from the piezoelectric substrate, $k$ is the rate reaction constant. 


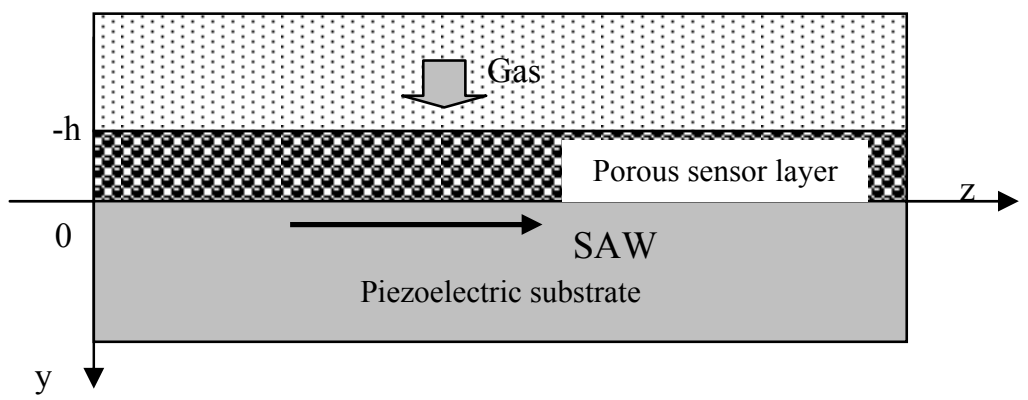

Figure 2: Model of a gas sensor with a sensitive layer on the piezoelectric substrate.

At steady-state conditions the general solution of the diffusion equation is:

$$
C_{A}=C_{1} \exp \left(y \sqrt{\frac{k}{D_{K}}}\right)+C_{2} \exp \left(-y \sqrt{\frac{k}{D_{K}}}\right)
$$

where $C_{1}$ and $C_{2}$ are integral constants.

At boundary conditions on the surface $(y=-h) \mathrm{C}_{\mathrm{A}}=\mathrm{C}_{\mathrm{As}}$ and $\partial C / \partial y=0$ the profile of the gas molecules concentration in the sensor layer is:

$$
C_{A}(y)=C_{A s} \frac{\cosh \left(|y| \sqrt{k / D_{k}}\right)}{\cosh \left(|-h| \sqrt{k / D_{k}}\right)}
$$

$C_{A s}$ is the target gas concentration outside the film on the surface $y=-h$. The concentration profile depends on the thickness of the sensor layer and the constants $k$ and $D_{K}$. Fig. 3 presents an example of the molecule gas concentration profile. Similar as in resistance sensors let us now assume that the electrical conductance $\sigma(y)$ of the sensor layer is linear to the gas concentration $C_{A}(y)$ [7]:

$$
\sigma(y)=\sigma_{0}\left(1 \pm a \cdot C_{A}(y)\right)
$$

where $\sigma_{0}$ is the initial layer conductance in the air, $a$ is the sensitivity coefficient and the sign \pm denotes the combination layer type conductivity and oxidation or reduction properties of the target gas. Experimental data for $\mathrm{H}_{2}$ have been presented by Sakai et al. [7], when $C_{A s}$ was fixed at $800 \mathrm{ppm}$ at a temperature of $350^{\circ} \mathrm{C}$, and assumed to be $a=1 \mathrm{ppm}^{-1}$. For these data the sensitivity data for $\mathrm{H}_{2}$ fit fairly well to the correlation line for $\sqrt{k / D_{K}}=0,01 \mathrm{~nm}^{-1}$.

The electrical conductance of the whole film is obtained by integrating $\sigma(y)$ over the whole range of y $(y=-h ; 0)$. That treatment has been proposed by Sakai, Williams and Hilger [7, 10]. A semiconductor layer in the SAW sensor cannot be treated in the same way.Because the profile of molecule gas concentration in a semiconducting sensor layer changes with the distance from the piezoelectric substrate (fig. 3), the acoustoelectric interaction differs in every sheet of the layer. In order to analyze such a sensor layer we assume that the film is a uniform stack of infinitesimally thin sheets (fig. 4) with a variable concentration of gas molecules and a different electric conductance. Each sub-layer is in 


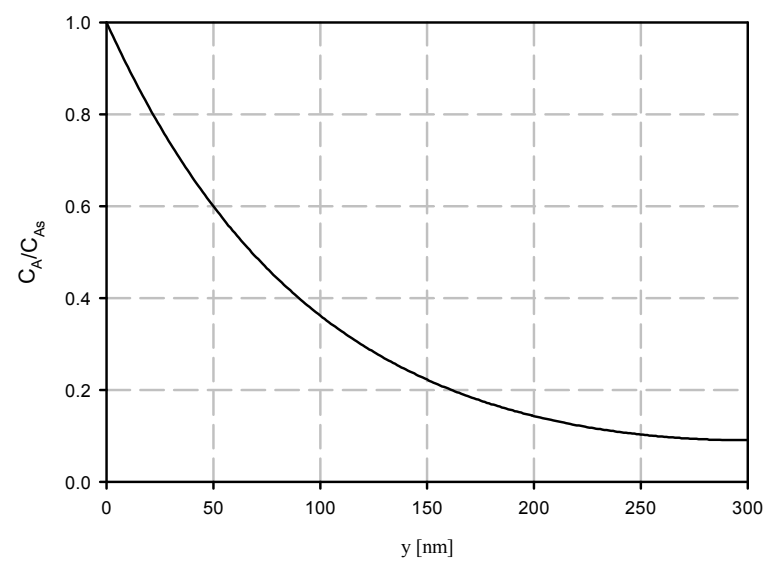

Figure 3: Gas concentration profile in a layer $300 \mathrm{~nm}$ thick and for $\sqrt{k / D_{K}}=0,01$.

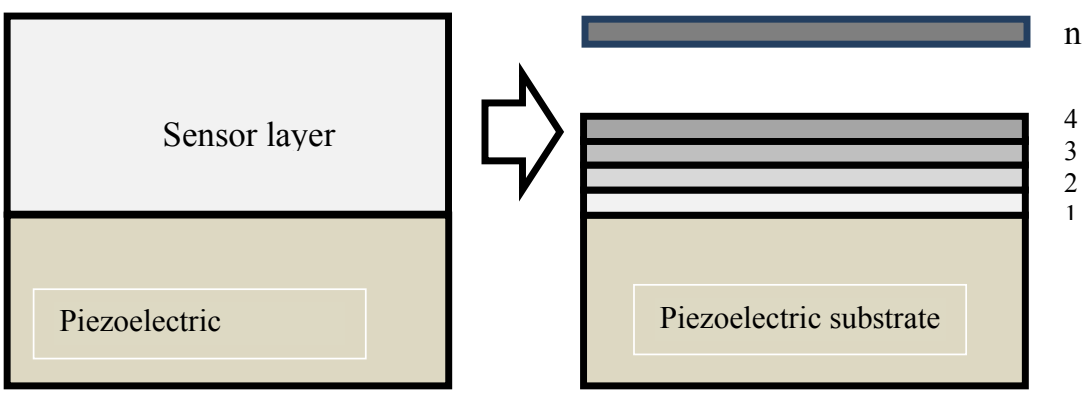

Figure 4: $\quad$ Model of a sensor layer divided into n-sub-layers.

another distance from the piezoelectric wave-guide. The schematic diagram in fig. 5 presents the way of analyzing the SAW gas sensor.

The resultant admittance is calculated basing on the impedance transformation law of each sub-layer to the surface of the wave guide. The surface conductance of the sub-layers is calculated applying the profile of concentration of the gas and Eq. (22). This resultant impedance applies the Ingebrigtsen formula to calculate changes in the propagation velocity of the surface wave. The transformation impedance law is derived from the general expressions for $\varphi$ ' and $\mathrm{D}_{\mathrm{y}}$ ' in the region $0>y>-h[5,11]$.

The normalized impedance at any arbitrary plane $y$ is [5]:

$$
z_{E}^{\prime}(y)=\frac{-i k_{0} \varepsilon_{0}\left(e^{k_{0} y}+\frac{B}{A} e^{-k_{0} y}\right) e^{-k_{0} y}}{-k_{0} \varepsilon_{0}\left(e^{k_{0} y}-\frac{B}{A} e^{-k_{0} y}\right) e^{-k_{0} y}}
$$




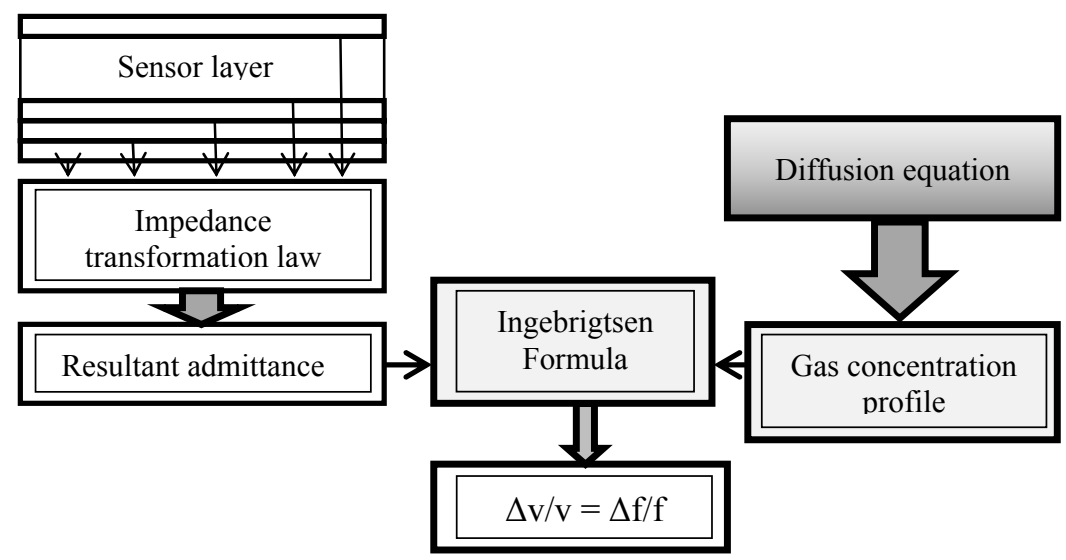

Figure 5: Analytical diagram of the SAW gas sensor.

The constant $B / A$ is evaluated by setting $y=-h$ in (23), and this gives the impedance transformation law for a single layer in the distance $-h$ :

$$
z_{E}^{\prime}(0)=\frac{i \cdot \tanh \left(k_{0} h\right)+z_{E}^{\prime}(-h)}{1-i \cdot z_{E}^{\prime}(-h) \cdot \tanh \left(k_{0} h\right)}
$$

It can be shown, that the Ingebrigtsen formula for $n$-sub-layers takes the following form [12]:

$$
\frac{\Delta v}{v_{0}}=-\operatorname{Re}\left\{\frac{\Delta k}{k_{0}}\right\}=-\frac{K^{2}}{2} \frac{\sigma_{T_{2}}^{2}\left(1 \pm a C_{A}\right)^{2}\left[1+\sum_{i=1}^{n-1} f\left(y_{i}, \sigma\left(y_{i}\right)\right)\right]^{2}}{\sigma_{T_{2}}^{2}\left(1 \pm a C_{A}\right)^{2}\left[1+\sum_{i=1}^{n-1} f\left(y_{i}, \sigma_{T_{2}}\right)\right]^{2}+\left[1+\sum_{i=1}^{n-1} g\left(y_{i}, \sigma\left(y_{i}\right)\right)\right]^{2}\left(v_{0} C_{S}\right)^{2}}
$$

where $n$ - number of sub-layers and $C_{S}=\varepsilon_{0}+\varepsilon_{p}^{T}, \sigma_{T_{2}}=\sigma_{T_{1}} \exp \left(\frac{E_{g}}{2 k} \cdot \frac{T_{2}-T_{1}}{T_{1} T_{2}}\right)$

$$
\begin{gathered}
\mathrm{T}_{1}=300 \mathrm{~K}, \sigma_{T_{1}}=\sigma_{0} \\
f\left(y_{i}, \sigma_{C S}\right)=\sum_{i=1}^{n-1} \frac{1-[\tanh (k y)]^{2}}{[1+\tanh (k y)]^{2}+\left[\tanh (k y) \cdot \frac{\sigma_{C S}}{\varepsilon_{0} v_{0}}\right]^{2}} \\
g\left(y_{i}, \sigma_{C S}\right)=\sum_{i=1}^{n-1} \frac{[1+\tanh (k y)]^{2}+\tanh (k y) \cdot\left(\frac{\sigma_{C S}}{\varepsilon_{0} v_{0}}\right)^{2}}{[1+\tanh (k y)]^{2}+\left[\tanh (k y) \cdot \frac{\sigma_{C S}}{\varepsilon_{0} v_{0}}\right]^{2}}
\end{gathered}
$$




\section{Numerical results of modelling a SAW gas sensor}

As an example of the adaptability of this theory the results of a numerical analysis have been presented. A thin layer of a semiconductor is placed on a Y-Z $\mathrm{LiNbO}_{3}$ waveguide. Results show the changes in the velocity of propagation depending on the concentration of the surrounding gas $\left(\mathrm{H}_{2}, \mathrm{CO}_{2}, \mathrm{NO}_{2}, \mathrm{NH}_{3}\right)$, the thickness of the semiconductor layer $\left(\mathrm{WO}_{3}\right)$, the pore radius and the sensor temperature. In the numerical analysis the following values of the involved constants have been assumed: $\sigma_{s}=v_{0} C_{S}=1.6 \times 10^{-8}\left[\Omega^{-1}\right]$, sensitivity coefficient $a=1$ $\left[\mathrm{ppm}^{-1}\right], \mathrm{E}_{\mathrm{g}}=2,7[\mathrm{eV}]$ for $\mathrm{WO}_{3}$ as for solid material [13].

Experimental results confirm the analytical model of the SAW sensor (Fig.8).

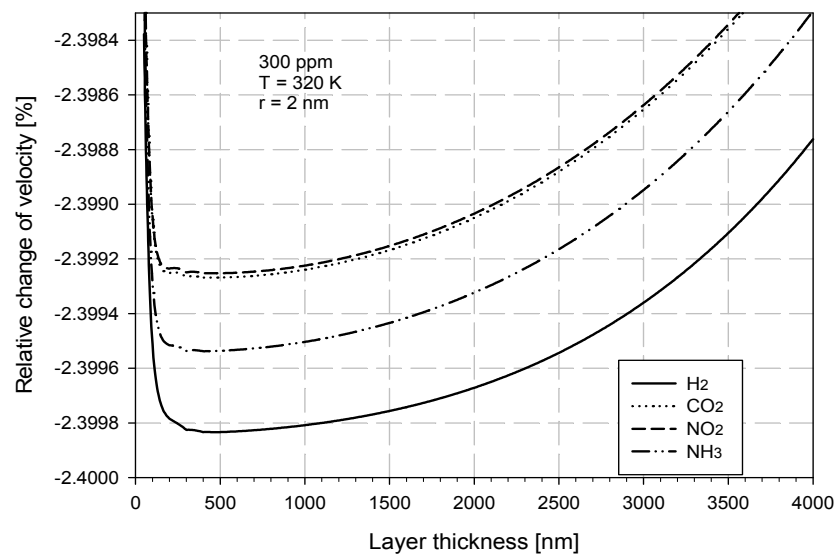

Figure 6: Changes of the SAW velocity propagation vs. the thickness of the $\mathrm{WO}_{3}$ sensor layer at $300 \mathrm{ppm} \mathrm{H}_{2}, \mathrm{CO}_{2}, \mathrm{NO}_{2}$ or $\mathrm{NH}_{3}$. Pore radius $\mathrm{r}=2 \mathrm{~nm}$.

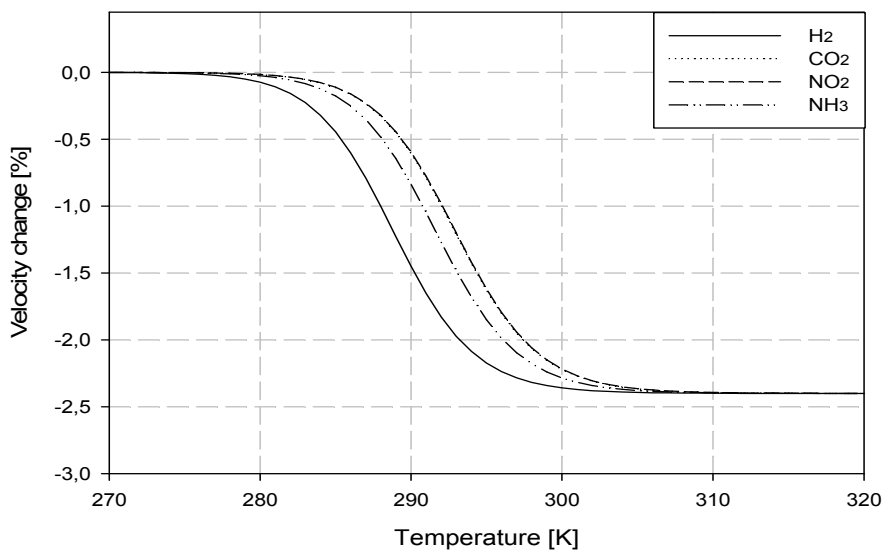

Figure 7: Changes of the SAW velocity propagation vs. the temperature of the $\mathrm{WO}_{3}$ sensor layer for $\mathrm{H}_{2}, \mathrm{CO}_{2}, \mathrm{NO}_{2}, \mathrm{NH}_{3}$ gases. 


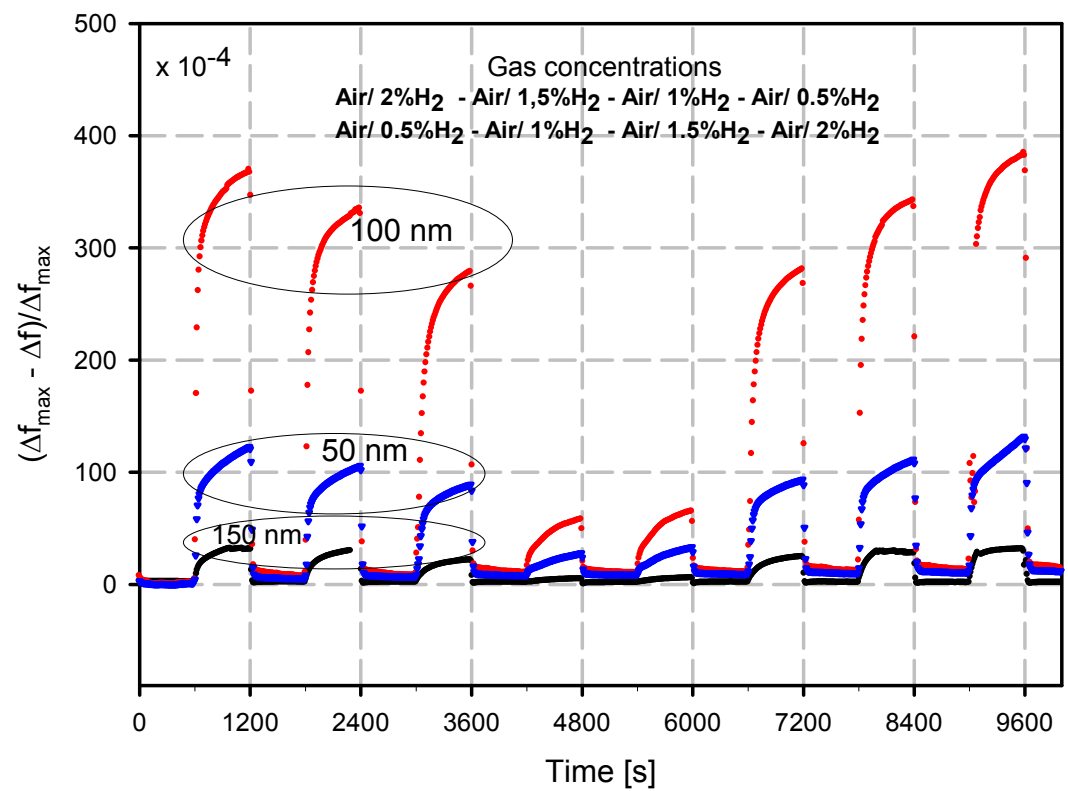

(a)

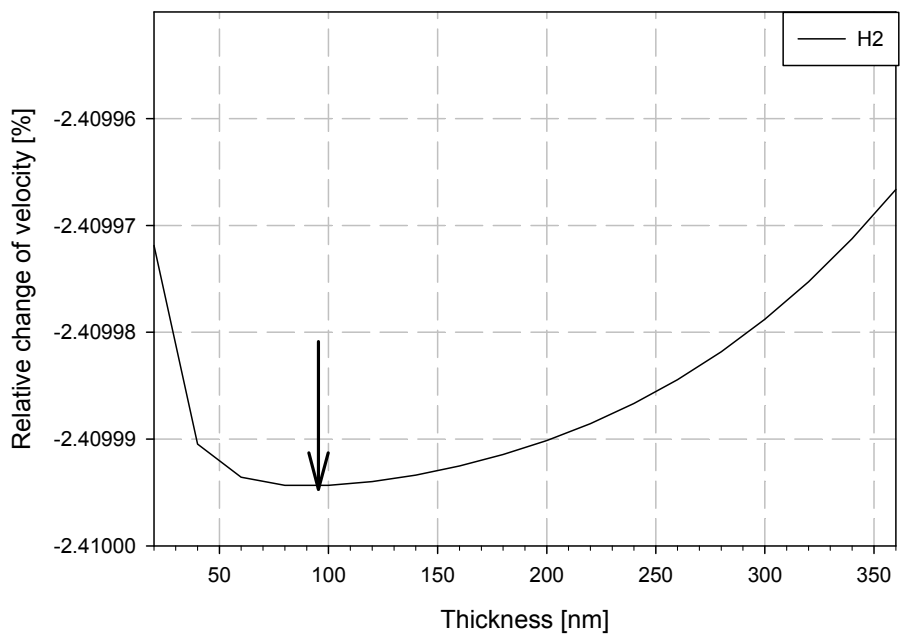

(b)

Figure 8: a. Experimental data concerning a $\mathrm{WO}_{3}$ layer with different thickness $(50,100,150 \mathrm{~nm})$. b. Numerical data, $0.5 \% \mathrm{H}_{2}$ gas. 
According to numerical results the optimal thickness of the sensor layer is about $96 \mathrm{~nm}$. Experimental results reach their maximum in a $100 \mathrm{~nm}$ thick layer.

\section{Conclusions}

In this paper a new analytical model of a $\mathrm{SAW}$ gas sensor and numerical results have been shown for the gases $\mathrm{H}_{2}, \mathrm{CO}_{2}, \mathrm{NO}_{2}, \mathrm{NH}_{3}$ and sensing layers $\mathrm{WO}_{3}$. The profile of the concentration of gas in the sensor layer has been applied in order to model the acoustoelectric effect in the SAW gas sensor. A porous semiconductor layer has been divided into sub-layers. The influence of the impedance above the piezoelectric substrate on the relative change of the SAW velocity has been calculated. In the sensor layer the Knudsen diffusion is assumed.

Analytical results are compatible with experimental results. The new analytical model may be used to optimize the structure of SAW gas sensors.

\section{Acknowledgement}

This work is financed by a grant from the Ministry of Science and Higher Education No. N N505 374237.

\section{References}

[1] W. Jakubik, M. Urbańczyk, E. Maciak, T. Pustelny, Bilayer structures of $\mathrm{NiO}_{\mathrm{x}}$ and PdIn Surface Acoustic Wave and Electrical gas sensor systems, Acta Phys. Pol. A, vol. 116, no 3, pp. 315-320, 2009.

[2] W. Jakubik, Investigation of thin film structure of $\mathrm{WO}_{3}$ and $\mathrm{WO}_{3}$ with $\mathrm{Pd}$ for hydrogen detection in a surface acoustic wave sensor system, Thin Solid Films515, pp. 8345-8350, 2007.

[3] G. Kino, T. Reeder, A Normal Mode Theory for the Rayleigh Wave Amplifier, IEEE Transaction on Electron Devices, Ed-18, 10, October 1971.

[4] W. Jakubik, M. Urbańczyk, The electrical and mass effect in gas sensors of the SAW type, J. Tech. Phys., 38(3), pp. 589-596, 1997.

[5] B.A. Auld, Acoustic Fields and Waves, vol. 2, J Willey and Sons, NY1973.

[6] D. L. White, Amplification of ultrasonic waves in piezoelectric semiconductors, J. Appl. Phys., Aug. 1962.

[7] Go Sakai, Naoki Matsunaga, Engo Shimanoe, Noboru Yamazone, Theory of gas-diffusion controlled sensitivity for thin film semiconductor gas sensor. Sensors and Actuators, B 80, pp. 125-131, 2001.

[8] K.M. Lakin, H.J. Shaw, Surface Wave Delay Line Amplifiers, IEEE Trans. MTT-17, pp. 912-920, 1969.

[9] J. W. Gardner, A non - linear diffusion - reaction model of electrical conduction in semiconductor gas sensors, Sensors and Actuators, B1, pp. 166-170, 1990.

[10] D. E. Williams, A. Hilger, Solid state gas sensors, Bristol, 1987. 
[11] T. Hejczyk, M. Urbańczyk, W. Jakubik, Semiconductor sensor layer in SAW gas sensors configuration, Acta Phys. Pol. A, 118(6), pp. 1153-1157, 2010.

[12] T. Hejczyk, M. Urbańczyk, W. Jakubik, Analytical model of semiconductor sensor layers in SAW gas sensors, Acta Phys. Pol. A, 118 (6), pp. 11481152, 2010.

[13] J. Portier, H.S. Hilal, Thermodynamic correlations and band gap calculations in metal oxides, Progress in Solid State Chemistry, 32, pp. 207-217, 2004. 